\title{
Detection of low frequency variants of the NLRP3 gene in "mutation- negative" CAPS patients using massive parallel sequencing
}

\author{
J Thiem* ${ }^{*}$, M Lesche $^{2}$, A Dahl $^{2}$, A Kränkel $^{2}$, J Roesler ${ }^{1}$, A Rösen-Wolff ${ }^{1}$ \\ From 8th International Congress of Familial Mediterranean Fever and Systemic Autoinflammatory Diseases \\ Dresden, Germany. 30 September - 3 October 2015
}

\begin{abstract}
Introduction
Recent studies showed a notable frequency of somatic mosaicism of the NLRP3 gene in chronic, infantile, neurological, cutaneous and articular (CINCA) syndrome (35\%) and Muckle-Wells syndrome (MWS)(12.5\%) patients that were not detectable by common Sanger sequencing.
\end{abstract}

\section{Objectives}

We are currently trying to detect and quantify low frequency NLRP3 variants in mutation-negative patients, who suffer from a CINCA Syndrome, MWS or Familial Cold Autoinflammatory Syndrome (FCAS) or show cryopyrin-associated periodic syndrome (CAPS)-like symptoms without a classical phenotype.

\section{Methods}

The exons of the NLRP3 gene were amplified via PCR from genomic PBMC DNA. The obtained PCR products were sequenced with an Illumina HiSeq platform. For SNP calling we used the GATK pipeline of the 1000 Genome Project, if the coverage attained 40,000 fold. In order to prove the accuracy of the method, we quantified dilutions of a known heterozygous mutation (T348M) mixed with wildtype DNA. For the correlation between the test results and the phenotype of the patients we developed a survey including symptoms and medical treatment.

\section{Results}

In one CINCA patient we detected a new NLRP3 variant (L359S) in $30 \%$ of the sequence. Using a cut-off of $5 \%$

'University Hospital Carl Gustav Carus, Department of Pediatrics, Dresden, Germany

Full list of author information is available at the end of the article mutated DNA sequences, we did not detect any other mutation of the NLRP3 gene in the other 47 samples we tested so far. We tried to increase the sensitivity by establishing a new statistic method $(\mathrm{M}+2 \mathrm{SD})$, setting the cut-off at $0.5 \%$. This led to a drastic reduction of specificity with irreproducible results.

\section{Conclusion}

Massive parallel sequencing is a reliable method for the quantification of low frequency variants of the NLRP3 gene. Increasing the sensitivity ( $<5 \%$ cut-off) results in detecting PCR artifacts and a dramatic loss of specificity. The probability of somatic mosaicism in mutation-negative CAPS patients is higher if the symptoms are more severe. Although $35 \%$ of mutation-negative CINCA patients and $12.5 \%$ of mutation-negative MWS patients harbor somatic mutations, this seems to be extremely rare in patients with CAPS-like symptoms without classical CAPS phenotype.

\section{Acknowledgements}

This study was supported by Novartis Pharma GmbH, Nürnberg (ARW); BMBF Projekt NGSgoesHPC (ML) and SFB655 (DFG) (AD, AK).

\footnotetext{
Authors' details

${ }^{1}$ University Hospital Carl Gustav Carus, Department of Pediatrics, Dresden, Germany. ${ }^{2}$ BIOTEC TU Dresden, Deep Sequencing Group SFB655, Dresden, Germany.
}

Published: 28 September 2015

doi:10.1186/1546-0096-13-S1-P34

Cite this article as: Thiem et al:: Detection of low frequency variants of the NLRP3 gene in "mutation- negative" CAPS patients using massive parallel sequencing. Pediatric Rheumatology 2015 13(Suppl 1):P34.
C Biomed Central

(c) 2015 Thiem et al. This is an Open Access article distributed under the terms of the Creative Commons Attribution License (http:// creativecommons.org/licenses/by/4.0), which permits unrestricted use, distribution, and reproduction in any medium, provided the original work is properly cited. The Creative Commons Public Domain Dedication waiver (http://creativecommons.org/publicdomain/ zero/1.0/) applies to the data made available in this article, unless otherwise stated. 